\title{
Revista Brasileira de Ciências do Envelhecimento Humano: produção científica de 2004 a 2010
}

\author{
Silvana Alba Scortegagna*, Ana Carolina Bertoletti De Marchi", Camila Pereira Leguisamo"**,

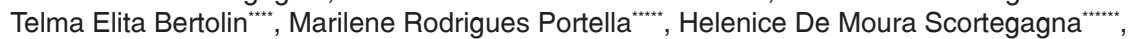 \\ Eliane Lucia Colussi ${ }^{* * * * * *}$, Regiane Boff"
}

\section{Resumo}

O objetivo deste estudo foi investigar a produção científica da Revista Brasileira de Ciências do Envelhecimento Humano, entre os anos de 2004 e 2010. Foram analisados 16 volumes, totalizando 184 publicações, com base nos seguintes critérios: quantidade de artigos em cada número de revista; origem geográfica; natureza da autoria; gênero dos autores; distribuição por tema de estudo e tipo de trabalho. Os dados demonstraram que o número de volumes publicados aumentou a partir de 2009, e que a revista manteve a regularização de sua periodicidade. A região Sul foi a que apresentou maior número de trabalhos. Houve predominância da autoria feminina, seguida de autoria múltipla. A área do conhecimento predominante foi ciências da saúde com temas relacionados à saúde pública, materializados por profissionais da Fisioterapia. Na sequência, destaca-se a participação da psicologia, da enfermagem e da educação com temas de pesquisas variados. Este trabalho atingiu os objetivos propostos e contribuiu para gerar diretrizes para o incentivo de publicações de outras áreas do conhecimento, novas temáticas de investigação e formatos de pesquisa.

Palavras-chave: Bibliometria. Envelhecimento. Periódicos.

* Psicóloga. Docente do Programa de Pós-Graduação Stricto Sensu em Envelhecimento Humano, Universidade de Passo Fundo, Campus I - Bairro São José - BR 285 Km 17, CEP 99052-900 - Cx. Postal 611 - Passo Fundo -RS, Tel: (54) 3316-8384.E-mail: silvanalba@upf.br

** Bacharel em Informática. Docente do Programa de Pós-Graduação Stricto Sensu em Envelhecimento Humano - Universidade de Passo Fundo.

**** $\quad$ Fisioterapeuta. Docente do Programa de Pós-Graduação Stricto Sensu em Envelhecimento Humano Universidade de Passo Fundo.

***** Bióloga. Docente do Programa de Pós-Graduação Stricto Sensu em Envelhecimento Humano - Universidade de Passo Fundo.

****** $\quad$ Enfermeira. Docente do Programa de Pós-Graduação Stricto Sensu em Envelhecimento Humano - Universidade de Passo Fundo.

Enfermeira. Docente do Programa de Pós-Graduação Stricto Sensu em Envelhecimento Humano - Universidade de Passo Fundo.

Licenciada em História. Docente do Programa de Pós-Graduação Stricto Sensu em Envelhecimento Humano - Universidade de Passo Fundo.

Acadêmica do curso de Psicologia da Universidade de Passo Fundo.

$\hookrightarrow_{\rightarrow}$ http://dx.doi.org/10.5335/rbceh.2013.2515 


\section{Introdução}

A produção científica em periódicos científicos visa à disseminação de conhecimentos. Pode ser considerada condição básica para a evolução da ciência. Historicamente, além de retratar os principais fatos do desenvolvimento científico, o periódico científico pode ser considerado um espaço institucional, pois se insere no universo das comunicações formais das inúmeras atividades científicas conduzidas nas diferentes áreas.

No campo do envelhecimento humano, por exemplo, ainda são escassos os periódicos que se dedicam a publicar artigos de nível técnico-científico. Entre aqueles que contribuem para 0 crescimento dessa área, encontram-se: a) Revista Geriatria \& Gerontologia, da Sociedade Brasileira de Geriatria e Gerontologia (SBGG); b) Revista Amazonense de Geriatria e Gerontologia, vinculada à Universidade do Estado do Amazonas; c) Revista Kairós, vinculada ao Programa de Estudos Pós-Graduados em Gerontologia, da Pontifícia Universidade Católica de São Paulo; d) Revista Brasileira de Geriatria e Gerontologia (anteriormente, Textos sobre Envelhecimento); e) Revista Brasileira de Ciências do Envelhecimento Humano (RBCEH), vinculada ao Programa Stricto Sensu em Envelhecimento da Universidade de Passo Fundo; f) Revista de Estudos Interdisciplinares Sobre o Envelhecimento, vinculada à Universidade Federal do Rio Grande do Sul.

Nesse cenário, a RBCEH é a única publicada no interior do estado do Rio Grande do Sul. Historicamente, em sua primeira edição, em 2004, a RBCEH nasce junto com a proposta de implantar um curso de mestrado interdisciplinar em Ciências do Envelhecimento Humano, na Universidade de Passo Fundo. Para levar adiante essa proposta, consolidada em 2007, houve a necessidade de ampliar a produção científica sobre o envelhecimento e de reunir as investigações e as experiências de outras instituições de ensino superior, comprometidas com a área gerontológica e geriátrica. A revista passa, então, a ser um novo veículo dos acontecimentos científicos, culturais e éticos em torno das questões interdisciplinares do envelhecimento, de forma aberta e crítica; ao mesmo tempo em que contribui para a melhoria das condições de vida dos idosos.

Inserida, portanto, no grupo dos periódicos nacionais, a revista foi classificada como B3, no ano de 2012, de acordo com a avaliação da Qualis. Essa categorização diz respeito à estratificação da qualidade da produção intelectual dos programas de pós-graduação, que subsidia a avaliação dos programas credenciados pela instituição, conduzida pela Coordenação de Aperfeiçoamento de Pessoal de Nível Superior (Capes) (YAMAMOTO et al., 2002).

Além disso, desde 2009, a revista integra o Scientific Electronic Library Online (SciELO), um modelo para a publicação eletrônica cooperativa de periódicos, especialmente desenvolvido para responder às necessidades da comunicação científica nos países em desenvolvimento e, particularmente, na América Latina e Caribe. Busca contribuir para o desenvolvimento da 
pesquisa científica nacional e regional, pelo aperfeiçoamento e ampliação dos meios de disseminação, publicação e avaliação dos seus resultados, fazendo uso intensivo da publicação eletrônica.

Quanto à disseminação e ao armazenamento das produções científicas comunicadas, a RBCEH opera no formato impresso e online, proporcionando acesso aberto aos artigos publicados. Desse modo, acolhe textos representativos de estudos, verificações e experiências que promovem o desenvolvimento humano em sua fase tardia, e outras que se revestem de novas possibilidades para o envelhecimento em toda a sua extensão.

A missão da revista é publicar artigos inéditos de pesquisa e desenvolvimento, que representem contribuição efetiva para essa área do conhecimento, no âmbito interdisciplinar. Entre as publicações que atualmente figuram nesse periódico, quais sejam, artigos de revisão, de investigação, relatos de casos clínicos, resumos de teses, observa-se que ainda não consta nenhum estudo que contemple a análise do próprio periódico.

Os primeiros estudos sobre avaliação de periódicos científicos foram realizados com revistas médicas latino-americanas na década de 1960 (FERREIRA; KRZYZANOWSKI, 2003). A partir daí, são várias as instituições nas diversas áreas do conhecimento que desenvolvem esses trabalhos. Entre os pesquisadores, é de comum acordo que a análise da produção científica em áreas específicas do conhecimento, por meio de revisões sistemáticas da literatura e de pesquisa bibliométrica, é importante para o avanço do saber
(MELO-SILVA; LEAL; FRACALOZZI, 2010; NORONHA e AMBIEL, 2006). Tal prática permite um olhar distanciado sobre o desenvolvimento científico da área, identificando lacunas e temas que requerem mais conhecimento científico e pesquisas.

Tendo em vista que a avaliação dos periódicos é o principal instrumento para garantir um padrão mínimo de qualidade nas publicações, que as revistas científicas são responsáveis pelo registro da produção intelectual e dos avanços no campo do conhecimento (COSTA; YAMAMOTO, 2008), e que não há registros de análise da $\mathrm{RBCEH}$, parece compreensível que esse veículo de comunicação seja alvo de estudo. Nesse contexto, o presente trabalho busca investigar a produção científica publicada na RBCEH, no período de 2004 a 2010.

\section{Método}

Trata-se de uma pesquisa descritiva analítica, operacionalizada por meio da observação direta dos artigos publicados na íntegra, nas versões impressas do periódico. Foram analisados 16 volumes da RBCEH da Universidade de Passo Fundo, totalizando 184 artigos, publicados no período de 2004 a 2010.

Os artigos foram divididos por ano e subdivididos por volume e número. Para o levantamento e análise dos dados, utilizaram-se alguns critérios considerados relevantes pelas autoras e estabelecidos por Witter (1999). Os itens pesquisados foram: (a) a quantidade de volumes e o total de publicações por ano; (b) a distribuição da produção por região 
geográfica (Sul, Sudeste, Norte, Nordeste, Centro-Oeste, parcerias regionais, internacionais e parcerias nacionais e internacionais); (c) a natureza da autoria (individual ou múltipla); (d) o gênero e a formação dos autores. Os dados obtidos foram analisados quantitativamente por meio de frequência e percentual.

\section{Resultados e discussão}

Inicialmente, levantou-se a quantidade de volumes e números e o total de artigos publicados por ano. Os resultados desse levantamento estão apresentados na Tabela 1.

Tabela 1. Distribuição geral da quantidade de volumes e números, bem como do total de artigos publicados por ano $(\mathrm{N}=184)$

\begin{tabular}{|c|c|c|c|c|}
\hline Ano & Volume & Número & $\begin{array}{l}\text { Total de artigos } \\
\text { publicados por número }\end{array}$ & $\begin{array}{c}\text { Total de artigos } \\
\text { publicados por ano }\end{array}$ \\
\hline \multirow[t]{2}{*}{2004} & 1 & 1 & 10 & 18 \\
\hline & 1 & 2 & 8 & \\
\hline \multirow[t]{2}{*}{2005} & 2 & 1 & 8 & 16 \\
\hline & 2 & 2 & 8 & \\
\hline \multirow[t]{2}{*}{2006} & 3 & 1 & 10 & 21 \\
\hline & 3 & 2 & 11 & \\
\hline \multirow[t]{2}{*}{2007} & 4 & 1 & 12 & 25 \\
\hline & 4 & 2 & 13 & \\
\hline \multirow[t]{2}{*}{2008} & 5 & 1 & 13 & 26 \\
\hline & 5 & 2 & 13 & \\
\hline \multirow[t]{3}{*}{2009} & 6 & 1 & 13 & 39 \\
\hline & 6 & 2 & 13 & \\
\hline & 6 & 3 & 13 & \\
\hline \multirow[t]{3}{*}{2010} & 7 & 1 & 13 & 39 \\
\hline & 7 & 2 & 13 & \\
\hline & 7 & 3 & 13 & \\
\hline
\end{tabular}

Observa-se que a partir do ano de 2009 houve um aumento no número de artigos publicados anualmente na RBCEH ( $\mathrm{n}=39$ ), com o acréscimo de um terceiro volume. Esse resultado, além de acompanhar a tendência dos periódicos dos Estados Unidos e da América Latina (HILL, 2004), deriva das exigências para a inserção da revista no SciELO.
Entretanto, já em 2007, houve um aumento de textos publicados por número $(n=13)$. Parece que esse incremento decorre de vários aspectos. De maneira geral, nota-se, nesse período, um crescimento expressivo da produção brasileira devido ao incremento dos programas de pós-graduação, do número de pós-graduandos e titulados, bem como da 
melhor qualificação do corpo docente das universidades. (DE MEIS; ARRUDA; GUIMARÃES, 2007). Aí também se insere a consolidação do Mestrado em Envelhecimento Humano da Universidade de Passo Fundo.

Outro aspecto a ser considerado é o de que, nesse contexto, começa a emergir uma compreensão mais efetiva de que, para se abarcar as complexidades do mundo e desvendar o conhecimento, necessita-se de análises mais integradas, que abordem diversas dimensões, uma vez que a realidade é multifacetada. Como desdobramento, surge a demanda de um movimento em favor da interdisciplinaridade.

Embora o conceito de interdisciplinaridade tenha surgido no século $\mathrm{XX}$, somente a partir da década de 1960 começa a ser enfatizado como necessidade de transcender o conhecimento fragmentado (MINAYO, 1991). Tal construto pode ser entendido como uma das formas de compreensão do mundo por meio de uma relação cooperativa, de reciprocidade, entre duas ou mais disciplinas. Isso, por sua vez, exige determinadas características de personalidade dos sujeitos envolvidos como flexibilidade, confiança e sensibilidade (FAZENDA, 1996; SANTOMÉ, 1998).

Para avaliar a procedência dos artigos, verificou-se a produção por origem geográfica. Assim, avaliou-se a região, o país e as parcerias regionais e internacionais estabelecidas. Esses resultados encontram-se na Tabela 2.
Tabela 2. Distribuição da produção por origem $(\mathrm{N}=184)$

\begin{tabular}{lrr}
\hline \multicolumn{1}{c}{ Origem } & Frequência & \multicolumn{1}{c}{$\%$} \\
\hline Sul & 115 & 62,5 \\
Sudeste & 44 & 23,9 \\
Nordeste & 13 & 7,1 \\
Centro-Oeste & 4 & 2,2 \\
Internacional & 7 & 3,8 \\
Norte & 1 & 0,5 \\
Total & 184 & 100,0 \\
\hline
\end{tabular}

Os resultados quanto à origem da produção indicaram que a região Sul foi a que mais contribuiu para as publicações da revista $(\mathrm{n}=115 ; 62,5 \%)$, seguida pela região Sudeste $(\mathrm{n}=44 ; 23,9 \%)$. A região Norte, por sua vez, foi a que menos contribuiu $(\mathrm{n}=01 ; 0,5 \%)$. Salienta-se que a editoração da revista está localizada na região Sul, o que pode ter influenciado nesse resultado. Além disso, a posição dos principais estados deve ser analisada com base na distribuição dos grupos de pesquisa e pesquisadores mestres e doutores.

No censo de 2006, dos grupos de pesquisa no Brasil (CNPQ, 2012), os cinco primeiros estados de maior destaque em produção científica, dentre os $21.024(67,8 \%)$ grupos brasileiros e $65.515(69,1 \%)$ pesquisadores doutores, foram: São Paulo (27\% do total de grupos e $30,4 \%$ de doutores); Rio de Janeiro (13,2\% de grupos e $13,4 \%$ de doutores); Rio Grande do Sul (10,4\% de grupos e 9,5\% de doutores); Minas Gerais (9,1\% de grupos e 8,8\% de doutores); e Paraná ( $8,1 \%$ de grupos e $8,8 \%$ de doutores). Considerando que a RBCEH está vinculada a um programa de pós-graduação do interior do estado do Rio Grande do Sul, justificam-se as estimativas reveladas no presente estudo. A Tabela 3 apresenta a frequência das publicações por área do conhecimento e formação dos autores. 
Tabela 3. Distribuição das frequências das publicações por área do conhecimento e formação dos autores $(\mathrm{N}=184)$

\begin{tabular}{|c|c|c|c|}
\hline Áreas do conhecimento & Áreas específicas & Frequência & $\%$ \\
\hline \multicolumn{4}{|l|}{ Ciências Humanas } \\
\hline & Psicologia & 33 & 17,9 \\
\hline & Educação & 12 & 6,5 \\
\hline & Sociologia & 3 & 1,6 \\
\hline & Filosofia & 3 & 1,6 \\
\hline & Letras & 3 & 1,6 \\
\hline & Geografia & 1 & 0,5 \\
\hline & História & 1 & 0,5 \\
\hline \multicolumn{4}{|l|}{ Ciências da Saúde } \\
\hline & Fisioterapia & 36 & 19,5 \\
\hline & Enfermagem & 30 & 16,3 \\
\hline & Educação Física & 21 & 11,4 \\
\hline & Odontologia & 4 & 2,2 \\
\hline & Medicina & 6 & 3,2 \\
\hline & Terapia Ocupacional & 3 & 1,6 \\
\hline & Fonoaudiologia & 5 & 2,7 \\
\hline & Farmácia & 3 & 1,6 \\
\hline & Nutrição & 3 & 1,6 \\
\hline \multicolumn{4}{|l|}{ Ciências Sociais Aplicadas } \\
\hline & Direito & 5 & 2,7 \\
\hline & Serviço Social & 2 & 1,1 \\
\hline & Administração & 1 & 0,5 \\
\hline \multicolumn{4}{|l|}{ Linguística, Letras e Artes } \\
\hline & Artes & 3 & 1,6 \\
\hline \multicolumn{4}{|l|}{ Engenharias } \\
\hline & Engenharia Civil & 1 & 0,5 \\
\hline & Engenharia Agronômica & 1 & 0,5 \\
\hline \multicolumn{4}{|l|}{ Ciências Biológicas } \\
\hline & Ciências Biológicas & 2 & 1,1 \\
\hline Total & & 184 & 100,0 \\
\hline
\end{tabular}

Conforme evidenciam os dados da Tabela 3, Ciências da Saúde foi a área do conhecimento mais evidente $(n=111$; $60,3 \%$ ), consolidada por artigos relacionados à saúde pública, desenvolvidos por profissionais da Fisioterapia $(n=36$;
19,5\%). A saúde pública tem como objeto de estudo a saúde e a doença no seu âmbito social, aborda as relações biológicas e sociaise e as expressões emocionais e afetivas. Nesse cenário, a proposta interdisciplinar coloca-se como exigência 
interna, uma vez que há um ilimitado campo de possibilidades a ser explorado, e uma ligação direta com o mundo vivido, o mundo do sofrimento, da dor e da morte (MINAYO, 1991).

$\mathrm{Na}$ sequência, emergem textos de profissionais da Psicologia $(\mathrm{n}=33$; $17,9 \%)$, Enfermagem $(\mathrm{n}=30 ; 16,3 \%)$ e Educação Física $(\mathrm{n}=21 ; 11,4 \%)$. As Ciências Humanas também desempenharam papel importante nas pesquisas relativas à temática. Entre os estudos, verifica-se a predominância da pesquisa sobre doenças crônicas e degenerativas e síndromes geriátricas. Frequentemen- te, essas patologias envolvem hábitos, comportamentos, estilos de vida. Esses resultados também foram constatados em outros estudos (PRADO; SAYD, 2004) e demonstram a preocupação com a biologia do envelhecimento na população idosa. Tradicionalmente, o envelhecimento populacional associou uma carga maior de doenças crônico-degenerativas, mais incapacidades e aumento do uso de serviços de saúde (LUBITZ et al., 2003). O Quadro 1 apresenta a distribuição das publicações por ano, gênero dos autores, natureza da autoria, área do conhecimento e tipo de trabalho.

Quadro 1. Distribuição dos artigos publicados por ano, gênero dos autores, natureza, área do conhecimento e tipo de trabalho

\begin{tabular}{|c|c|c|c|c|c|c|c|c|c|c|c|c|c|c|c|}
\hline \multirow[t]{2}{*}{ Ano } & \multirow[t]{2}{*}{ v. } & \multirow[t]{2}{*}{ n. } & $\begin{array}{c}\text { Total de } \\
\text { artigos } \\
\text { publicados }\end{array}$ & \multirow[t]{2}{*}{$\begin{array}{c}\text { Total de } \\
\text { publicações } \\
\text { por ano }\end{array}$} & \multicolumn{2}{|c|}{$\begin{array}{c}\text { Gênero } \\
\text { dos auto- } \\
\text { res }\end{array}$} & \multicolumn{2}{|c|}{$\begin{array}{c}\text { Natureza } \\
\text { da autoria }\end{array}$} & \multicolumn{5}{|c|}{ Área do conhecimento } & \multicolumn{2}{|c|}{$\begin{array}{l}\text { Distribuição por tipo } \\
\text { de trabalho }\end{array}$} \\
\hline & & & & & M & $\mathrm{F}$ & Mul. & Ind. & CS & $\mathrm{CH}$ & CSA & LLA & E CB & $\begin{array}{l}\text { Manuscrito } \\
\text { Teórico }\end{array}$ & $\begin{array}{l}\text { Relato de } \\
\text { pesquisa } \\
\text { de campo }\end{array}$ \\
\hline \multirow[t]{2}{*}{2004} & 1 & 1 & 10 & 18 & 5 & 14 & 6 & 4 & 5 & 4 & 1 & & & 6 & 4 \\
\hline & 1 & 2 & 8 & & 9 & 8 & 6 & 2 & 4 & 4 & & & & 4 & 4 \\
\hline \multirow[t]{2}{*}{2005} & 2 & 1 & 8 & 16 & 6 & 14 & 5 & 3 & 3 & 4 & & & 1 & 2 & 6 \\
\hline & 2 & 2 & 8 & & 2 & 18 & 7 & 1 & 4 & 4 & & & & 3 & 5 \\
\hline \multirow[t]{2}{*}{2006} & 3 & 1 & 10 & 21 & 4 & 19 & 8 & 2 & 4 & 5 & 1 & & & 6 & 4 \\
\hline & 3 & 2 & 11 & & 9 & 17 & 8 & 3 & 6 & 4 & 1 & & & 6 & 5 \\
\hline \multirow[t]{2}{*}{2007} & 4 & 1 & 12 & 25 & 8 & 20 & 7 & 5 & 5 & 6 & & & 1 & 5 & 6 \\
\hline & 4 & 2 & 13 & & 11 & 21 & 9 & 4 & 8 & 2 & 1 & & & 6 & 7 \\
\hline \multirow[t]{2}{*}{2008} & 5 & 1 & 13 & 26 & 7 & 37 & 12 & 1 & 6 & 6 & 1 & & & 7 & 7 \\
\hline & 5 & 2 & 13 & & 17 & 23 & 13 & - & 9 & 1 & 1 & & 2 & 5 & 8 \\
\hline \multirow[t]{3}{*}{2009} & 6 & 1 & 13 & 39 & 9 & 30 & 11 & 2 & 6 & 4 & 1 & 2 & & 5 & 8 \\
\hline & 6 & 2 & 13 & & 8 & 42 & 12 & 1 & 10 & 2 & & 1 & & 5 & 8 \\
\hline & 6 & 3 & 13 & & 4 & 31 & 12 & 1 & 8 & 5 & & & & 5 & 8 \\
\hline \multirow[t]{3}{*}{2010} & 7 & 1 & 13 & 39 & 17 & 29 & 12 & 1 & 11 & 1 & 1 & & & 6 & 7 \\
\hline & 7 & 2 & 13 & & 5 & 32 & 12 & 1 & 11 & 2 & & & & 5 & 8 \\
\hline & 7 & 3 & 13 & & 11 & 27 & 11 & 2 & 11 & 2 & & & & 4 & 9 \\
\hline Total & 16 & & 184 & 184 & 132 & 382 & 151 & 33 & 111 & 56 & 8 & 3 & 22 & 80 & 104 \\
\hline
\end{tabular}


Os resultados demonstraram que há um número maior de publicações envolvendo mulheres $(\mathrm{n}=382 ; 74,3 \%)$, seguido pela parceria entre homens e mulheres $(\mathrm{n}=151 ; 29,3 \%)$ e de apenas homens $(\mathrm{n}=$ $132 ; 25,6 \%$ ). A superioridade feminina na publicação de trabalhos em contraponto com uma minoria masculina parece encontrar justificativa na crescente inserção institucionalizada de mulheres em grupos de pesquisa, o que veio a alterar a configuração eminentemente masculina do campo científico (FERREIRA et al., 2008). Para esses autores, as mulheres atuaram decisivamente sobre o processo de institucionalização em curso no pós-Segunda Guerra Mundial, caracterizado como um momento de transição de um padrão de atividade restritivo para um padrão profissional moderno, baseado em oportunidades mais amplas de acesso e na estruturação de uma carreira.

No que se refere à análise por tipo de trabalho, buscou-se investigar a modalidade do manuscrito. Para tanto, consideraram-se as seguintes categorias: relato de pesquisa de campo, relato de pesquisa documental ou manuscrito teórico. Os resultados evidenciaram que a maior parte do material publicado refere-se a relatos de pesquisa de campo $(n=104$; $56,5 \%)$. Outros estudos de Oliveira et al. (2006), Suehiro et al. (2007) e Suehiro e Rueda (2009) também confirmam essa predominância. Neste trabalho, tal evidência pode ser justificada pela natureza das pesquisas publicadas relacionadas às Ciências da Saúde e Humanas, áreas de maior predominância.

\section{Considerações finais}

O objetivo deste estudo foi realizar um levantamento dos textos publicados na RBCEH, desde 2004 até 2010, com a intenção de apresentar um panorama de suas publicações e seu desenvolvimento. Para tanto, foram consideradas algumas categorias que auxiliaram a elucidar a quantidade de artigos publicados por número da revista, a região em que prevaleceram as pesquisas, a natureza da autoria, gênero dos autores, a distribuição por tema de estudo e por tipo de trabalho.

Os resultados revelaram que a $\mathrm{RB}$ $\mathrm{CEH}$ manteve seu crescimento, principalmente com o aumento do número de publicações a partir de 2009. Paralelamente, confirmou-se a predominância da região Sul como a mais produtiva e com maior representatividade nos trabalhos com autoria feminina e múltipla.

De maneira geral, os resultados obtidos reforçam a importância da realização de pesquisas com o objetivo de caracterizar as publicações de periódicos científicos, e avaliar a qualidade do conteúdo publicado. Isso, por sua vez, garante a emergência de diretrizes capazes de indicar novos temas, formatos de pesquisas e auxiliar na melhor distribuição de fomentos.

Por fim, pesquisas desse porte são relevantes não apenas por possibilitarem o desenho e o desenvolvimento dos periódicos científicos, mas, sobretudo, por indicarem as lacunas que devem ser preenchidas. Trazem, também, contribuições à disseminação, à qualidade e ao progresso do conhecimento produzido nas 
academias, o qual, cada vez mais, exige relações entre várias disciplinas. Além disso, o foco na produção e na divulgação alerta para uma preocupação essencial no fazer científico atual, que se traduz sobre as formas de produção e os veículos de divulgação científica no Brasil.

\section{Brazilian Journal of Human Aging Sciences: Scientific production from 2004 to 2010}

\section{Abstract}

The objective of this study was to investigate the scientific production of the Brazilian Journal of Human Aging Sciences between 2004 and 2010. Sixteen volumes were analyzed, totaling 184 publications, based on the following criteria: Number of articles in each journal's issue, geographic origin, authorship nature, author gender, distribution by study theme and kind of work. The data showed that the number of published volumes increased from 2009 and the journal kept the regularity of its periodicity. The southern region was the one which presented the biggest number of works. There was a predominance of female authorship, followed by multiple authorship. The predominant knowledge area was the health sciences with themes related to public health, carried out by physiotherapy professionals. Following these, there is a highlight in the participation of psychology, nursing and education with varied research themes. This paper has achieved the proposed aims and contributed for the generation of guidelines for the incentive of publications in other areas of knowledge, new themes for investigation and research formats.

Keywords: Bibliometry. Aging. Journals.

\section{Referências}

COSTA, A. L. F.; YAMAMOTO, O. H. Publicação e avaliação de periódicos científicos: paradoxos da avaliação qualis de psicologia. Psicologia em Estudo [online], Maringá, v. 13 , n. 1, p. 13-24, 2008.

CNPq - CONSELHO NACIONAL DE DESENVOLVIMENTO CIENTÍFICO E TECNOLÓGICO. Séries históricas. Diretório dos Grupos de Pesquisa no Brasil, Brasília, Disponível em: <http://dgp.cnpq.br/censos/ series_historicas/index_basicas.htm $>$. Acesso em: jun. 2012.

DE MEIS, L.; ARRUDA, A. P.; GUIMARÃES, J. The Impact of Science in Brazil. IUBMB Life, Malden, v. 59, n. 4-5, p. 227-234, maio 2007.

FAZENDA, I. C. A. Integração e interdisciplinaridade no ensino brasileiro: efetividade ou ideologia? 4. ed. São Paulo: Loyola, 1996.

FERREIRA, M. C. G.; KRZYZANOWSKI, R. F. Periódicos científicos: critérios de qualidade. Pesquisas de Odontologia Brasileira, São Paulo, v. 17, p. 43-48, 2003.

FERREIRA, L. O. et al. Institucionalização das ciências, sistema de gênero e produção científica no Brasil (1939-1969). História Ciências Saúde-Manguinhos [online], v. 15, suppl., p. 43-71, 2008.

HILL, D. L. Latin American shows rapid rise in published Science \& Engineering articles. National Science Foundation, Arlington, ago. 2004. Disponível em: <http://nsf.gov/ statistics/infbrief/nsf04336/nsf04336.pdf>. Acesso em: 10 jun. 2012.

LUBITZ J. et al. Health, life expectancy, and health care spending among the elderly. $N$ Engl J Med, Waltham, v. 349, n. 11, p. 104855 , set. 2003.

MELO-SILVA, L. L.; LEAL, M. S.; FRACALOZZI, N. M. N. Produção científica em congressos brasileiros de orientação vocacional e profissional: período 1999-2009. Revista 
Brasileira de Orientação Profissional, São Paulo, v. 11, n. 1, p. 107-120, 2010.

MINAYO, M. C. S. Interdisciplinaridade: uma questão que atravessa o saber, o poder e o mundo vivido. Medicina, Ribeirão Preto, v. 24 , n. 2 , p. $70-7,1991$.

NORONHA, A. P. P.; AMBIEL, R. A. M. Orientação profissional e vocacional: análise da produção científica. Psico-USF, São Paulo, v. 11, n. 1, p. 75-84, 2006.

OLIVEIRA, K. L. et al. Produção científica de 10 anos da revista Psicologia Escolar e Educacional (1996/2005). Psicologia Escolar e Educacional, São Paulo, v. 10, n. 1, p. 283292, 2006.

PRADO, S. D.; SAYD, J. D. A pesquisa sobre envelhecimento humano no Brasil: pesquisadores, temas e tendências. Ciência \& Saúde Coletiva, Rio de Janeiro, v. 9, n. 3, p. 763-772, 2004.

SANTOMÉ, J. T. Globalização e interdisciplinaridade: o currículo integrado. Porto Alegre: Artmed, 1998.

SUEHIRO, A. C. B.; RUEDA, F. J. M. Revista Avaliação Psicológica: um estudo da produção científica de 2002 a 2007. Avaliação Psicológica, São Paulo, v. 8, n. 1, p. 131-139, 2009.

SUEHIRO, A. C. B. et al. Produção científica da revista Psico-USF de 1996 a 2006. Psico-USF, São Paulo, v. 12, n. 2, p. 327-334, 2007.

WITTER, G. P. Metaciência e leitura. Em G. P. Witter (Org.). Leitura: textos e pesquisa, Campinas: Alínea, p. 13-22, 1999.

YAMAMOTO, O. H. et al. Avaliação de periódicos científicos brasileiros da área de psicologia. Ciência da Informação, Brasília, v. 31 , p. $163-177,2002$. 